\title{
La question migratoire dans la chanson française actuelle : trois études de cas
}

\author{
Audrey COUDEVYLLE-VUE (Valenciennes) ${ }^{1}$
}

\section{Summary}

While documentaries, films, and plays are currently examining the humanitarian tragedy of mass exodus from former Mesopotamia and parts of sub-Saharan Africa, what can be said about the French popular song? After P. Perret, G. Manset, A. Souchon, B. Lavilliers, and J. Clerc, who dealt with migration and exile between the end of the 1980s and the first decade of the new millenium, who are the songwriters and singers that evoke the tragedies of today's migrants? Do they have sufficient visibility in the French song? Which musical genres are chosen to represent them? The present paper tries to establish a brief panorama of the migration issue in the current French song by analysing a number of fundamentally different songs, such as "La ballade des clandestins" by Arthur H (Soleil dedans, 2014), "El dulce de leche" adapted by L.E.J. (En attendant l'album, 2015), and "Grand dérèglement" by Frànçois and the Atlas Mountains (Solide mirage, 2017).

Des abris insalubres de la jungle de Calais aux bulldozers procédant à leur démantèlement ; des familles entassées dans les camps établis le long de la clôture barbelée de la frontière hongroise aux errances des migrants subsahariens, proies de passeurs libyens esclavagistes ; du périple meurtrier d'enfants, tel le petit Syrien Aylan, symbole de tous ces autres bambins anonymes noyés à celui plus chanceux de nouveaux-nés poussant leur premier cri sur des navires de secours ; de ces embarcations de fortune bondées et chavirantes, aux pérégrinations de port en port de l'Aquarius, contenant plus de 629 individus en situation de crise sanitaire ; de ces hommes perdus en mer aux cadavres rejetés sur diverses plages européennes : journaux télévisés, papiers ou numériques ont relayé presque quotidiennement, depuis 2010, le sort tragique des migrants.

Ces images instantanées ont été massivement récupérées par le traitement politique. En surface, chacun sait que ces problématiques migratoires relèvent du politique ou des politiques publiques, dont la finalité est la gestion des affaires de la cité. Mais, si la question de l'immigration sature l'agenda politique des démocraties européennes comme l'horizon 
médiatique, quelle visibilité les migrants ont-ils en dehors de cette actualité ? Qu'en est-il de leur prise en charge notamment artistique et culturelle ? Alors que documentaires, fictions, pièces de théâtre et festivals ${ }^{2} s^{\prime}$ emparent avec plus ou moins de succès ou de confidentialité de la question migratoire, quelle audibilité la chanson confere-t-elle à ceux que l'on nomme sans distinction 'sans-papiers `, clandestins, refugiés ou migrants ? Ne pourraient-ils trouver un accueil singulier, poétique et éthique, dans le creuset d'une chanson elle-même par nature d'essence migratoire ? La chanson ne disposerait-elle pas également de sa propre vérité politique, qui plus est lorsqu'elle est véhiculée par les exilés eux-mêmes, à l'image d'un Montand (alias Ivo Livi) ayant fui l'Italie fasciste, d'un Aznavour exilé de l'Europe centrale arménienne, ou d'un Corneille enfant rescapé du génocide Rwandais.

La chanson française n'a jamais semblé en reste pour soulever le sort de ces exilés : Pierre Perret et "Lily ", Gérard Manset et "Avant l'exil " ", Alain Souchon avec "C'est déjà ça ", Bernard Lavilliers avec "Exil " ${ }^{6}$ puis " Exilés »", Julien Clerc avec " Réfugiés " ${ }^{8}$ les ont célébrés. Mais ces chansons ont toutes pour particularité d'avoir été écrites, composées et interprétées entre la fin des années quatre-vingt - il y a donc déjà plus de 35 ans -, les années quatre-vingt-dix et la première décennie des années 2000 , soit bien avant notre vaste crise migratoire. Qui depuis lors (j’entends quel interprète - si possible influent - de la scène française) évoque la tragédie actuelle vécue par ces migrants ? Quelle chanson peut également avoir aujourd'hui autant de rayonnement qu'en eut «Lily » de Pierre Perret, toujours régulièrement reprise ?? Plusieurs autres questions encore méritent d'être soulevées : Que chante-t-on des conditions des migrants, de leur statut, de leur périple ? Comment les interprètes actuels rendent-ils compte de leur situation? Quels choix ou perspectives artistiques ou esthétiques adoptent-ils?

Par le biais de l'analyse de deux chansons relevant de l'actualité récente, puisque créées et enregistrées respectivement en 2014 et 2017, à l'acmé de la crise migratoire ("La ballade des clandestins " d'Arthur $\mathrm{H}$ et "Grand dérèglement " du groupe Frànçois and the Atlas Mountain), $j$ 'interrogerai les $<$ intentionnalités ${ }^{10}$ et les thématiques dans lesquels s'inscrivent ces productions chansonnières. J'aimerais encore proposer quelques analyses concernant l'un des succès du trio L.E.J., leur reprise de «El dulce de leche » dans En attendant l'album en 2015. Certes, le cadre narratologique de cette chanson ne réfere pas à la même situation migratoire que celle évoquée dans les deux premières chansons, orientées en filigrane du côté du drame se jouant en Méditerranée. Le contexte général de l'exil dans «El dulce de leche " est celui d'une émigration politique massive des Chiliens, après le coup d'État militaire le 11 septembre 1973 du Général Pinochet mettant fin au régime démocratique de Salvador Allende. Les conditions d'exode sont bien sûr très différentes, néanmoins, la chanson a ceci d'intéressant qu'elle n'interroge plus seulement les difficultés d'un parcours ou d'une traversée (ici inexistante pourrait-on dire puisqu'il s'est agi d'atteindre l'Europe en avion), mais met en lumière l'، après trajet । : le sort des exilés dans un pays qui n'est pas le leur et qui sont contraints d'y rester, de s'y faire une place. 
Pour finir quant à la justification de notre corpus, ces trois chansons ont également pour mérite, par leurs choix esthétiques variés, de s'emparer de la question migratoire hors des formes du rap, du slam ou du reggae, plus attendues sur la question.

\section{«La ballade des clandestins » ou une vision poético-romantique de l'exil}

Cette chanson de l'auteur-compositeur-interprète Arthur H est extraite de l'album Soleil dedans, sorti en septembre 2014 et enregistré à Montréal au Québec. Cette «ballade », dont le titre laisserait envisager un ancrage dans l'écho lointain d'une autre ballade, celle " des gens heureux $»^{11}$, offre une vision poético-romantique de l'exil où le périple des migrants s'effacerait au profit d'une vision du voyage comme le prolongement d'un accomplissement amoureux :

Tout près de rien, à côté de nulle part

Suivant de près quelques traces qui s'effacent

Les clandestins glissent vers la lumière

À mourir pour mourir autant mourir debout

Pars avec moi vers ce qui nous éclaire

On travaillera chez l'ami de ton frère

Les clandestins glissent vers la lumière

À mourir pour mourir autant mourir debout

Oh tu es fou malade mais je reste avec toi

Ne change pas c'est comme ça que je t'aime

Oh que je t'aime

Elle vient de loin et elle sait comment faire

Même tout en bas elle peut voir la lumière mais

Quand il l'embrasse elle se fout de tout

À mourir pour mourir autant mourir debout

Lui c'est obscur son cœur est un bunker

Il croit qu'il est toujours seul sur la terre mais

Quand elle l'embrasse il se fout de tout

À mourir pour mourir autant mourir debout

Oh tu es fou malade mais je reste avec toi

$\mathrm{Ne}$ change pas c'est comme ça que je t'aime 
Oh tu es fou malade mais je reste avec toi

Ne change pas c'est comme ça que je t'aime

Que je t'aime.

En effet, dans cette chanson il est frappant de constater que le traitement de la question migratoire prend un tour très particulier. Certes la première strophe installe un horizon d'attente saturé de lieux communs, mais propre à rendre compte de la nature même de la notion de clandestinité (« tout près de rien [...] nulle part [...] traces qui s'effacent »), dont les propriétés principales sont la dissimulation et l'invisibilité. Les clandestins sont ceux qui se soustraient, par nécessité, aux représentants d'une autorité en place, qui vivent en marge des lois, et dont on nie l'existence. Ils sont des ‘ riens > qu'il ne faut pas voir, qui n'ont pas leur place à côtés des nôtres. Ils sont pour finir ceux qui se sont embarqués, non sans péril, vers d'autres destins perçus potentiellement par eux-mêmes comme plus « lumineux `. Or, cette seule allusion à la lumière, en fin de troisième vers ( les clandestins glissent vers la lumière »), recevable également dans le sens mystique de "mourir ", mais en vue de renaître, va conférer à l'ensemble de la chanson une dimension et une ouverture complètement nouvelles. Ce seul mot ‘ lumière > va biffer toute référence à la mort. Même la réduplication, à quatre reprises, du vers "À mourir pour mourir autant mourir debout ", lui-même saturé dans sa construction par le martèlement du verbe ‘ mourir `, souligne davantage l'espoir, la recherche de la dignité que de la fin de soi.

Qui plus est, cette répétition particulière de l'expression « à mourir pour mourir " n'est pas anodine. Elle participe d'un maillage intertextuel intéressant faisant écho en premier lieu à l'un des premiers succès de Barbara, "À mourir pour mourir " ${ }^{12}$. Le « savoir mourir >, l'un des principes chers à l'artiste, n'est plus ici revendiqué comme condition de conservation de l'amour-propre, mais comme condition de conservation d'une humanité, se définissant par sa capacité même à aimer. D'ailleurs, cette perspective mortifere est à nouveau biffée par une autre réminiscence intertextuelle, intervenant immédiatement dans la strophe suivante, celle d'un des succès de Johnny Halliday cette fois : "Que je t'aime " ${ }^{13}$. Il semble désormais évident que, par le détachement appuyé de ces deux déclaratives en fin de strophe ("Oh que je t'aime »), Arthur $\mathrm{H}$ vise ici une chanson humaniste qui dépasserait la seule situation des migrants.

Ainsi, dès cette première strophe, c’est subrepticement toute la chanson qui " glisse vers la lumière ", vers la célébration de la liberté et de l'espace infini. Arthur H, jouant également dans le titre de l'homonymie du terme ‘ ballade , confere à sa chanson une tonalité éminemment poétique et narrative. Il envisage, tel un conteur, le périple de ces deux clandestins anonymes comme une échappée étincelante, certes périlleuse, mais protégée par les auspices de l'amour et de l'espérance, d'ailleurs essentiellement portées par la figure féminine. ${ }^{14}$ Transformé en une sorte de Pythie ancestrale ("Elle vient de loin et elle sait comment faire / Même tout en bas elle peut voir la lumière "), le personnage féminin semble plus aérien, radieux et dans l'acceptation de la folie que le personnage masculin, pour sa part sombre, 
solitaire et pragmatique ( Lui c'est obscur son cœur est un bunker / Il croit qu' il est toujours seul sur la terre "; " On travaillera chez l'ami de ton frère »).

Néanmoins, le personnage masculin finit lui aussi par succomber rapidement à cet état d'abandon amoureux ("Quand elle l'embrasse, il se fout de tout / À mourir pour mourir / autant mourir debout "). Ce basculement vers la fable amoureuse est d'autant mieux mis en valeur que le refrain ("Oh tu es fou malade mais je reste avec toi / Ne change pas c'est comme ça que je t'aime ») est accompagné par les envolées éblouissantes de la pedal-steel gui$\operatorname{tare}^{15} \mathrm{du}$ Canadien Joe Grass. Ces clandestins ne sont définitivement plus de pauvres hères mais des figures amoureuses majestueuses et dignes, prêtes certes à " marcher droit " (et pas seulement tout droit) mais aussi à " mourir debout".

De son propre aveu, Arthur $\mathrm{H}$ a voulu conférer à cette chanson les accents d'une ballade à la Johnny Cash. Revenant sur la conception des « clandestins ", il raconte :

J'étais à Big Sur [au sud de San Francisco], chez un vieux hippie qui écoutait la musique à fond la caisse. Ces sons qui arrivaient dans ce jardin luxuriant m’ont donné envie d'écrire une ballade à la Johnny Cash, une sorte de chronique contemporaine. ${ }^{16}$

Il rajoute encore que son objectif n'était pas tant d'écrire une chanson sur la condition misérable des migrants, que de rappeler qu'ils étaient aussi, malgré leur terrible destin, des êtres humains, avec des états d'âme, des histoires d'amour et des joies parfois. "La ballade des clandestins » aurait aussi trouvé son propos à la suite d'un voyage à Haïti où Arthur $\mathrm{H}$, accompagné de Nicolas Repac ${ }^{17}$, a été frappé de constater que ces habitants, pourtant ravagés par la pauvreté et la dévastation, vivaient des ` vies normales `:

Quand on a découvert Haïti avec Nicolas Repac, se souvient-il, on s'attendait à un monde dévasté à la Mad Max. On a rencontré des gens qui ont des vies normales et ne supportent plus d'être vus sous l'angle misérabiliste. Cela n'enlève rien à la misère extrême, mais cela sort de l'hystérie médiatique. La chanson va dans ce sens. (Campion 2014)

Ainsi, qualifiant lui-même son album de "poésie punk-mystique, sexuelle et chimérique " (Arthur H 2015), «La ballade des clandestins » construit définitivement une légende amoureuse plus qu'elle ne dénonce les conditions d'existence misérables de deux clandestins. La chanson, en ce sens, sublime le sujet migrant, non plus seulement perçu comme un laissé-pour-compte, à l'instar des figures développées dans les chansons de Francis Lalanne " Ouvrir son cœur » (2009) ou de Christophe Maé « Lampedusa " (2016), mais un humain avec des préoccupations, des besoins autres que ceux d'une survie primaire mais nécessaire.

La proposition artistique de la deuxième chanson étudiée, "Grand dérèglement ", aux influences pop-rock anglo-saxonnes et multiculturelles, bien qu'ayant également des velléités poétiques, semble s'inscrire davantage dans des intentions plus politiques. 


\section{"Grand dérèglement " ${ }^{18}$ (Frànçois and the Atlas Mountains) : une chanson politique?}

Avant d'analyser cette chanson, il faut rappeler qu'usuellement le traitement ' politique , de la figure de l'immigré, de l'immigration et des faits de société qui en découlent, demeurait surtout le fait, sur la scène française, du hip-hop, du rap ${ }^{19}$, mais encore de groupes aux influences musicales issues du reggae. Tryo ${ }^{20}, \mathrm{Zebda}^{21}$, Dub Inc ${ }^{22}$ ont pris en charge cette question pour en souligner les revendications identitaires et sociales. Plus récemment encore, le groupe de rock, à la poésie subtile, Debout sur le Zinc, pilier de la scène indépendante française, signe, après vingt ans de carrière, leur neuvième album, Eldorado(s), sorti en septembre 2015, opus engagé, comme en témoigne la chanson "Lampedusa " ${ }^{23}$, revenant sur les drames de l'immigration clandestine.

Pourquoi dès lors, choisir de traiter d'une chanson au rayonnement encore intime, "Grand dérèglement ", du groupe non moins discret Frànçois and the Atlas Mountains ? Probablement pour la raison que sans ‘ rugissements `, ni formules convenues, Frànçois and the Atlas Mountains, offre à la nouvelle pop française, aux influences indie pop ${ }^{24}$, la possibilité de s'inscrire dans le sillon de la chanson > engagée > d'une façon singulière, par le biais d'une certaine douceur, en partie entretenue par la voix ciselée du chanteur François Marry rappelant le lyrisme de Dominique A :

Dit s'y estimer content

D'encore dégager quelques excédents

Eux sillonnent le continent de long en large

Recherchent une place dans le sens de la marche

L'essence du pays échappe

L'histoire la rattrape, l'exode les happe

S'étale sur plusieurs années

Au milieu avale la Méditerranée

Combien de temps?

D'années, d'années?

Se laisser glisser

Dans ces dérèglements

Grand et grand dérèglement

Pauvres frappant nos deux mains

Sur l'algorithme des mots

En rythme pour demain

À voir avec quelle cadence

Ces rapports souffleurs nonchalance,

Va croire aux boniments 
De devins qui disent qu'un divin

Calcule nos régiments

Ils respirent comme ils mentent

Combien de temps?

D'années, d'années?

Se laisser glisser

Dans ces dérèglements

Grand et grand dérèglement.

«Grand dérèglement » évoque très précisément dès la première strophe la question migratoire. La chanson, extraite du quatrième album du groupe, Solide Mirage, sorti le 3 mars 2017 et produit sous le label Domino Records par Ash Workman (celui-là même qui produisit également Christine and The Queens), fait d'ailleurs référence, ainsi qu'en témoigne en interview François Marry, à la sombre année 2015, qui bouleversa l'artiste et l'amena à s'interroger :

C'est délicat de parler de politique dans des chansons, mais c'est inévitable quand tu écris dans des moments comme ceux-ci. J'ai une attirance pour l'abstraction, la poésie mais je me suis rendu compte que c'était devenue une technique d'autruche. Je ne comprends pas pourquoi les musiciens ne se posent pas plus la question de la responsabilité de leur parole. Et vue l'année 2015 qu'on s'est tous pris dans la tronche, il était nécessaire de se demander ce que l'on voulait dire. (Aïnouz 2017)

D'ailleurs, l'indéfinition nominative des migrants, désignés par l'unique pronom " eux ", témoigne bien que ces hommes, femmes et enfants qui traversent la Méditerranée en un flot humain, demeurent ceux que l'on expose aux yeux de tous, mais que surtout personne ne veut voir. Peut-être François Marry a-t-il finalement trouvé l'expression adéquate pour désigner ceux que la presse nomme sans distinction spécifique (migrants >, ( clandestins >, 〈 réfugiés `, ‘ sans-papiers > : Eux qui sillonnent le continent de long en large / Recherchent une place dans le sens de la marche ", comme un écho au " marcher droit » d'Arthur H.

La singularité de cette chanson, outre la voix aiguë et feutrée de son interprète (" épuisée ", précise François Marry, mais " pleine d'espoir et en recherche de clarté »; Guillaud 2017) tient aussi à la mixité des sonorités qu'elle propose. Alors que dès l'ouverture, "Grand dérèglement " s'inscrit sans conteste dans un style pop-rock anglo-saxon, la chanson bascule, aux premiers mots de François Marry, dans une subtile osmose composée d'harmonies celtiques et orientales. Les percussions de derbouka ouvrant la deuxième strophe consacrée à l'évocation de l'exode des migrants, succèdent aux riffs de guitares électriques, signature de l'indie pop. Genres musicaux, sonorités et rythmes du Monde se mélangent et se nourrissent entre eux.

Cette volonté d'imprégnation est également soutenue et revendiquée dans la construction du clip ${ }^{25}$ lui-même. Tourné dans l'enceinte du Palais de Justice de Bruxelles, on peut y 
observer les gracieuses déambulations et arabesques du danseur Mohammed Okal - ancien ambulancier palestinien à Gaza - qui effectue une danse traditionnelle au Moyen-Orient, le Dabké26. Du propre aveu du chanteur François Marry, filmer une Dabké dans un lieu aussi emblématique que le Palais de Justice de la capitale de l'Europe, " alors même que la chanson évoque les grands changements de notre monde, qui déplacent des populations et forcent les plus faibles à s'adapter à un mode de vie ardue et violent, est une manière d'attirer l'attention sur le côté positif de ces mutations " (Ménard 2017).

La symbolique entre ce lieu singulier et cette danse folklorique orientale, se renforce encore lorsque le danseur emmène dans son sillage le chanteur François Marry, et l'invite à former un duo. Leurs jetées de pas s'effectuent alors en miroir et rappellent, que cette Dabké n'est pas si éloignée dans son geste artistique de ce que peut être une danse celtique. Ce métissage chorégraphique parachève d'acheminer la chanson du côté d'un engagement accompli, reflet du discours empreint d'espérances de son interprète. Toutefois cet engagement - revendiqué par son auteur - tiendrait moins au contenu narré sur la question migratoire (somme toute assez consensuel voire convenu dans son propos) qu'au tissage interculturel, musical et artistique que la chanson propose. L'audibilité de ces deux pratiques musicales au départ distinctes, se muerait ici en une sorte de syncrétisme artistique et culturel qui, au lieu de fondre et de dissoudre chaque style entre eux, celtique et oriental, révèlerait aux contraires leur similitude.

\section{L.E.J. ou la culture du métissage}

Pour finir, $j$ 'aimerais clore mes analyses, quant au traitement de la question migratoire dans le panorama de l'actuelle chanson française, en dévoilant un dernier constat. Je n'ai jusqu'alors proposé ou cité que des chansons écrites ou interprétées par la gent masculine. Cette absence de voix ou de plumes féminines pour chanter ce sujet est-elle significative? Par comparaison, durant les années vingt et trente, les sorts des laissés-pour-compte, étaient essentiellement pris en charge par des interprètes féminines, en particulier les chanteuses « réalistes > telles que Damia, Fréhel ou Berthe Sylva, etc. Un peu plus tard, Édith Piaf interpréta également "Exodus ${ }^{27}$ en 1961, qui revient sur ce sujet. Mais, qu'en est-il aujourd'hui ?

À notre connaissance, il semble que seule l'auteur-compositeur-interprète Juliette, avec sa chanson "Aller sans retour " ${ }^{28}$, créée en 2008 , ait traité le sujet. L'actuelle scène française ne manque pourtant pas de talents féminins, ${ }^{29}$ encore moins d'auteures engagées ou perçues comme telles. ${ }^{30} \mathrm{Il}$ faudrait s'interroger - mais ce pourrait être l'objet d'un autre article - sur cette absence.

Toutefois, un véritable phénomène musical, grâce au succès de leurs mash-up $p^{31}$ Summer $2015^{32}$ et Summer 2016 33 , plébiscité par la presse du monde entier - internet en particulier et salué par les artistes influents du moment (David Guetta ou Pharell William qui l'accueillit pour sa première partie de concert en juin 2015) mérite notre attention. Le trio féminin L.E.J. ${ }^{34}$, deux voix et un violoncelle pour seuls instruments la plupart du temps, et sacré 
depuis février 2017 "meilleur révélation scène " aux $32^{\mathrm{e}}$ Victoires de la Musique, semble aussi avoir été sensible à la question de l'immigration, ainsi que le prouve leur reprise de «El Dulce de Leche $»^{35}$ du célèbre quatuor de reggae français, de la fin des années 90, Tryo ${ }^{36}$. Ce groupe eut d'ailleurs un rôle fondamental dans la découverte de L.E.J., puisqu'en 2013 ils lancent un concours de chant ouvert aux amateurs, que les trois étudiantes mélomanes remportent. Le trio est officiellement lancé. Ce parrainage originel, celui d'une formation connue autant pour ses engagements politiques et environnementaux que pour la singularité de son style musical, aux influences jamaïcaines, et ses choix scéniques dépouillés, valait bien d'être rappelé. Peut-être est-ce l'une des raisons pour lesquelles L.E.J. a repris «El Dulce de Leche» :

Il n'avait pas idée,

Il n'avait pas conscience,

À quel point lui manquait

Les terres de son enfance,

La Cordillère des Andes,

Les cocotes en papier,

Le fumé de la viande,

La confiture de lait.

Il n'avait pas idée,

On dira inconscience,

À quel point lui coûtait

D’être bloqué en France,

Rejoindre le pays,

L'odeur de l'orchidée,

Le temps n'a pas enfoui

El dulce de leche.

[Refrain]

Il faudra reprendre la route,

Devenir français coûte que coûte,

Réfugié dans un tiroir,

On passe le temps on garde espoir,

C'est ça être français j'en doute.

Il n'avait pas idée,

Il n'avait pas conscience,

Comme peuvent marquer

Les blessures de l'enfance,

Une larme à Paris, 
Une rose pour Pinochet,

Le temps n'a pas enfoui

Le palais d'Allende.

Comme il avait souri,

En parlant de la France,

Il raconta sa vie,

Ses années de silence,

Les tonnelles en fleur,

Le sang sur le pavé,

Le départ pour l'ailleurs,

El dulce de leche.

[Refrain]

Il faudra reprendre la route,

Devenir français coûte que coûte,

Réfugié dans un tiroir,

On passe le temps on garde espoir,

C'est ça être français sans doute,

El dulce de leche.

Il n'avait pas idée,

Mais c'est sans suffisance,

On ne peut qu'imaginer

Les parcours de l'errance,

Le prenant par la main,

Eh l'ami, on y est !

La famille les copains,

La confiture de lait.

Cette chanson, inspirée de l'exil contraint de Daniel Bravo, le percussionniste du groupe Tryo, né à Santiago du Chili en 1973, durant le coup d'État de Pinochet, arrivé à Bobigny à l'âge de 9 ans, sans maîtriser la langue, mêle la douceur nostalgique des souvenirs d'enfance - le goût de la confiture de lait - aux difficiles conditions d'existence des réfugiés. Sanspapiers, la vie d'exilés, pourtant espérée plus douce, reste précaire. Avant d'obtenir la nationalité française en 2007, Daniel Bravo s'est longtemps senti prisonnier sur le territoire, dans l'impossibilité de retourner au Chili (Beaudouin-Pilon 2010). La chanson a donc une résonance particulière et conforte l'idée d'un entre-deux, fait d'espérances et de déceptions, de nostalgie doucereuse et d'épreuves, dont les voix mêlées du trio L.E.J., couplées à la puissance et à la sobriété du violoncelle, rendent parfaitement compte. 
Outre le fait que cette chanson traite de notre thématique, elle présente surtout le double intérêt de mettre en lumière les différents métissages de la chanson comme l'éclectisme de ses interprètes. La formation classique des jeunes femmes (l'une a étudié au conservatoire de Saint-Denis et les deux autres ont suivi la formation de la Maîtrise Radio France) contrastant avec l'endroit où elles reçurent leurs enseignements, à Saint-Denis - ville populaire, réputée pour sa forte concentration d'immigrés, et qui voit émerger davantage de groupes de hip-hop ou de rap que de chanteuses potentiellement lyriques - appelle ici un autre métissage culturel : celui de genres artistiques diamétralement opposés (ou supposés l'être). Ce groupe rend donc poreuses les frontières entre deux formations et modes d'expression : l'une que l'on perçoit usuellement comme réservée à une élite sociale et intellectuelle, d'expression ' noble > et d'autres, issues de la rue, des quartiers populaires telles que le hip-hop, le rap ou le reggae.

L.E.J. revendique explicitement ce métissage : leur brillante reprise de "Seine SaintDenis Style ", écrite et composée par le slameur Grand Corps Malade, interprétée par Kool Shen et l'inénarrable Joey Star, anciens membres du groupe de rap NTM, rend hommage évidemment à leur ville mais également aux influences multiculturelles qui les ont nourries. Cette posture justifierait assurément leur titre de ` divas du macadam `.

Ainsi, grâce aux analyses de trois chansons actuelles, produites pourtant à l'acmé d'une tragédie humanitaire, il semblerait que la question migratoire puisse aussi devenir un objet de sublimation et que le traitement du migrant puisse sortir des clichés misérabilistes ou des figures convenues usuellement relayées à leur endroit, sans que pour autant rien ne soit minimisé ou omis du fléau qui les touche. Par le biais d'autres genres musicaux que ceux couramment ‘ légitimes ` pour déclamer leur sort, leur destinée est aussi envisagée avec poésie et grâce.

D'ailleurs, si de l'acmé d'une tragédie humanitaire on peut faire chanson, c'est que les espaces de l'entendement ne se laissent pas enfermer. Le tragique, par analogie de mouvement, migre d'un domaine vers un autre, les catégories de l'esprit accueillent les catégories politiques et poétiques dans le même entendement. Dans le règne des valeurs, il n'y a donc pas de porte idéologique. Tout est passage. Et c'est peut-être à partir de là, que la question migratoire doit commencer à trouver du sens dans le cœur des hommes. Ce n'est pas qu'une affaire de police mais une affaire d'humanisme, de liberté.

\section{Notes}

1 Audrey Coudevylle-Vue, Laboratoire CALHISTE, UVHC, France.

2 Le Festival d'Automne accueillait, entre début septembre et fin décembre 2016, dans plusieurs théâtres parisiens, la pièce 81 avenue Victor Hugo d'Olivier Coulon-Jablonka, créée en mai 2015. Celle-ci, née de la rencontre d'un collectif de migrants sans-papiers, qui occupaient l'Agence Pôle-Emploi désaffectée de l'avenue Victor Hugo dans le seizième arrondissement parisien, 
mettait en scène huit de ces membres racontant leur exil et leur parcours semé d'embûches. Mais, 81 avenue Victor Hugo rappelait également qu'au-delà de ces périples fous, à travers les pays et les mers, succédait l'implacable machine administrative française et ses exigences kafkaïennes. Au cinéma, le drame de Philippe Lioret, Welcome, avec Vincent Lindon, sorti en 2009, couronné d'une dizaine de récompenses, ou plus récemment encore la comédie dramatique finlandaise, cocasse et décalée, d'Aki Kaurismäki, sortie en mars 2017, L'autre côté de l'espoir, examinent dans des registres différents et par le biais de dispositifs variés, cette tragédie humanitaire. Rappelons encore le documentaire italien de Gianfranco Rossi, Fuocoammare, par-delà Lampedusa, sorti en septembre 2016 et Ours d'Or à la Berlinale la même année, dont le titre évoque tout à la fois une chanson sicilienne composée pendant la Seconde Guerre mondiale, "La mer en feu », comme la terrible réalité des migrants embarqués sur leurs esquifs.

3 De l'album Lily, sorti en 1977 ; paroles et musique Pierre Perret.

4 De l'album Matrice, sorti en octobre 1989 ; paroles et musique Gérard Manset.

5 Extrait de l'album éponyme, C'est déjà ça, sorti en octobre 1993 ; paroles d'Alain Souchon, musique de Laurent Voulzy.

6 Tirée de l'album Clair-obscur, sorti en 1997 ; paroles et musique de Guillaume Rossel et Bernard Lavilliers.

7 Extrait de l'album Causes perdues et musiques tropicales, sorti en 2010 ; paroles de Bernard Lavilliers, musique de Bernard Lavilliers et Bonga Kuenda.

8 De l'album Double enfance, sorti en 2005 ; paroles d'Étienne Roda-Gil, musique de Julien Clerc.

9 Julie B. Bonnie en 2012 et Les Ogres de Barback en 2005.

10 Terme utilisé par Ursula Mathis-Moser lors de sa conférence du 11 avril 2018 à l'Université d'Innsbruck.

11 «La ballade des gens heureux » (1975), paroles de Pierre Delanoë, musique et interprétation de Gérard Lenormand ; extraite de l'album Olympia 75. Un extrait explicite :

Tu n'as pas de titre ni de grade

Mais tu dis «tu " quand tu parles à dieu

Je viens te chanter la ballade

La ballade des gens heureux.

12 Dans Barbara chante Barbara (octobre 1945).

À mourir pour mourir

Je choisis l'âge tendre

Et partir pour partir

Je ne veux pas attendre

Je ne veux pas attendre

[...]

J'aime mieux m'en aller

Du temps que je suis belle

Qu'on ne me voit jamais

Fanée sous ma dentelle

Fanée sous ma dentelle. 
13 Paroles de Gilles Thibaut, musique de Jean Renard.

14 Le personnage de la femme est d'ailleurs au cœur de l'ensemble de l'album d'Arthur H comme d'autres titres peuvent en témoigner (p.ex. «Une femme qui pleure "; « La caissière du super »; "La femme étoile»).

15 Instrument à cordes, se rapprochant de la guitare mais de forme parallélépipédique, qui repose sur quatre pieds permettant au musicien de jouer assis. Un mécanisme permet de modifier le son des cordes par l'action de pédales. Les cordes peuvent être pincées par la main droite ou frottées par une barre d'acier (la steel bar) tenue par la main gauche. Ce type d'instrument est presque exclusivement utilisé dans la musique américaine.

16 Interview de décembre 2014, donnée à la veille de son concert au Rocher de Cenon (Aquitaine). Cf. Delneste 2014.

17 Guitariste et instrumentiste, Nicolas Répac a contribué à la composition de l'album L'or noir d'Arthur H, consacré à la poésie de la Caraïbe francophone. Il a également mis en musique L'or d'éros, album recueillant une sélection de textes et de poèmes sulfureux du $\mathrm{XX}^{\mathrm{e}}$ siècle mis en voix par Arthur H.

18 Paroles de François Marry, musique de François Marry, Amaury Ranger et Jean Thévenin ; extraite de l'album Solide mirage, enregistré en mars 2017, dans le Jet studio de Molenbeek à Bruxelles, Production Domino.

19 Le dernier album de l'emblématique groupe marseillais des années 90 IAM, Rêvolution, sorti en mars 2017, s'inscrit encore dans ces perspectives.

20 "El dulce de leche ", de l'album Ce que l'on sème (2008).

21 «Une vie de moins » de l'album Second tour, interprétée en octobre 2012 (paroles de Jean-Pierre Filiu), ou encore leur deuxième album Le bruit et l'odeur, déjà daté (1995), reprenant une phrase très controversée d'un des discours de Jacques Chirac en 1991, peuvent constituer des exemples probants.

22 Le groupe Dub Inc, porté par les voix de Bouchkour et Komlan, s'est imposé depuis dix ans comme fer de lance du reggae made in France. Mixant ce genre musical à des sonorités et rythmes kabyles ou plus largement à la world music, le groupe chante l'importance du mélange des styles, du métissage et du bien vivre ensemble. «Exil », extrait de l'album So What, sorti en 2016, est un hommage aux exilés qui ont contribué à bâtir l'Hexagone.

23 Enregistrée au studio Ferber en janvier 2015:

Quand t'a tenté ta chance à bord de ce bateau

T'imaginais la France comme un eldorado

Contre vents et marées, tu es resté debout

La Méditerranée avait une faim de loup, de loup

Est-ce que je serai le bienvenu chez vous

Est-ce que je serai le bienvenu c'est tout

Toi tu rêvais d'Europe

Quand sa mythologie

Gorgones et cyclopes

Restaient sourds à tes cris 
Maudits chants des sirènes

Leurs promesses de bonheur

Elles t'ont poussé sans peine

À surmonter ta peur, ta peur

Est-ce que je serai le bienvenu chez vous

Est-ce que je serai le bienvenu c'est tout

[...]

Lampedusa panse ses plaies au petit matin

Cette nuit la mort a frappé, moi je n'y pouvais rien

Lampedusa panse ses plaies au petit matin

Cette nuit la mort a frappé, moi

Moi j'étais trop loin.

24 Genre musical qui a émergé à la fin des années 80 , après l'effondrement du punk, du post punk et de la new wave. Il trouve ses rythmes et sonorités dans une pop davantage seventies.

25 Co-réalisé avec Jack Barraclough.

26 Signifiant ‘ coup de pied > en arabe, cette danse est effectuée lors de mariages ou de fêtes traditionnelles en Syrie, Palestine, Jordanie ou au Liban. Souvent improvisée, elle est menée par le ras (le chef de la danse) qui l'exécute en faisant tournoyer un collier de perles, le masbaha, tandis que les autres danseurs donnent le rythme. La dabké est soutenue par un chant d'accompagnement, lui-même improvisé. À ce chant s'ajoutent des instruments de musique comme la derbaké (ou derbouka, une percussion) ou des flûtes (meejwez).

27 Chanson écrite par Eddy Marnay, musique d'Ernest Gold :

Ils sont partis dans un soleil d'hiver

Ils sont partis courir la mer

Pour effacer la peur, pour écraser la peur

Que la vie a clouée au fond du cœur

Ils sont partis en croyant aux moissons

Du vieux pays de leurs chansons

Le cour chantant d'espoir

Le cœur hurlant d'espoir

Ils ont repris le chemin de leur mémoire.

28 Extraite de l'album Bijoux et babioles (2008) :

Ce que j'oublierai, c'est ma vie entière

La rue sous la pluie, le quartier désert

La maison qui dort, mon père et ma mère

Et les gens autour, noyés de misère

En partant d'ici, pour quel paradis ou pour quel enfer...

J'oublierai mon nom, j'oublierai ma ville

J'oublierai même que je pars pour l'exil

[...] 
Ce que j'oublierais, si j'étais l'un d'eux

Mais cette chanson n'est qu'un triste jeu

Et quand je les vois passer dans nos rues

Étranges étrangers, humanité nue

Quoi qu'ils aient fui

La faim, le fusil

Quoi qu'ils aient vendu

Je ne pense qu'à ce bout de couloir

Une valise posée en guise de mémoire...

29 Daphnée, Rose, Émilie Loiseau, Anaïs, Clarika, Berry, Émilie Simon, La Grande Sophie, Olivia Ruiz, Camille, Jane, Christine and The Queen, etc.

30 Zaz, Agnès Bihl voire un temps Pauline Croze, grâce à " Jeunesse affamée ", tirée de l'album Pauline Croze (2005).

31 Le fait de mélanger un, deux voire plusieurs titres sur une seule piste.

32 Dans En attendant l'album (août 2015).

33 Sorti le 4 août 2016. Mélange et reprise en un peu plus de 3 minutes seulement de 23 titres différents de Coldplay à Rihanna, en passant par Adèle ou Nekfeu.

$34 \mathrm{Du}$ nom de leurs trois jeunes interprètes : Lucie, Élisa, Juliette.

35 Écrite et composée par Christophe Mali, extraite de l'album Ce que l'on sème (2008).

36 Le groupe, composé de Christophe Petit, dit Mali, membre fondateur, Guizmo (Cyrile Célestin), Manu (Emmanuel Eveno), Danielito (Daniel Bravo) et Bibou (Sébastien Pujol) fut créé en 1995 et remporta de vifs succès essentiellement lors des années deux mille (p.ex. «L'hymne de nos campagnes "; "C'est du roots », en 2004 et surtout l'album Ce que l'on sème, sorti en 2008, disque de platine). Tryo revendique une approche engagée et politique de la musique (cf. l'album Grain de sable, en 2003, ou la chanson "Regardez-les ", 1998). Malgré leur rare apparition dans les médias, ils restent un groupe prisé du public.

\section{Bibliographie}

Aïnouz, Abigaïl : « Frànçois and the Atlas Mountains de retour avec un album politique Solide Mirage ». In : Les Inrockuptibles (12.01.2017), https://www.lesinrocks.com/2017/01/12/musique/ francois-the-atlas-mountains-de-retour-album-politique-solide-mirage-11901462/ (consultation 13.03.2017).

Arthur H : Le cauchemar merveilleux. Arles : Actes Sud, 2015.

Beaudouin-Pilon, Cybèle : "Parle-nous de ta toune : Tryo - Dulce de Leche ». In : Urbania (19.07.2010), https://urbania.ca/article/parle-nous-de-ta-toune-tryo-dulce-de-leche/ (consultation 18.12.2016).

Bolzman, Claudio : «De l'exil à la diaspora : l'exemple de la migration chilienne ». In : Fibbi, Rosita / Meyer, Jean-Baptiste (éds.) : Diasporas : développements et mondialisations. La Tour d'Aigues : 
L'Aube, 2002, http://horizon.documentation.ird.fr/exldoc/pleins_textes/pleins_textes_7/autrepart1/010028951.pdf (consultation 13.03.2017).

Campion, Alexis : «Rayonnantes envolées ». In : Le Journal du Dimanche (21.09.2014), http://www. lejdd.fr/Culture/Musique/Arthur-H-rayonnantes-envolees-688682 (consultation 07.01.2017).

Delneste, Yannick : «Arthur H: ‘ La musique est une espèce de fusée > ». In : Sud Ouest, Blog chanson C'est déjà ça (01.12.2014), http://chansonfrancaise.blogs.sudouest.fr/archive/2014/12/01/ arthur-h-1029817.html (consultation 07.01.2017).

Guillaud, Éric : "Frànçois and the Atlas Mountains, chanson française et pop anglo-saxonne en toute affinité (interview) ». In : France 3. Pays de la Loire (10.02.2017), https://france3-regions. francetvinfo.fr/pays-de-la-loire/loire-atlantique/nantes-metropole/nantes/interview-francoisand-the-atlas-mountains-chanson-francaise-pop-anglo-saxonne-toute-affinite-1 194047.html (consultation 13.03.2017).

Ménard, Laure : "Vidéo. Francofolies : l'interview 100\% Sud-Ouest de Frànçois and the Atlas Mountains ». In : Sud Ouest.fr La Rochelle (15.07.2017), https://www.sudouest.fr/2017/07/15/ video-francofolies-1-interview-100-sud-ouest-de-francois-the-atlas-mountains-3621252-722. php (consultation 31.03.2017).

\section{Discographie}

Arthur H : L'or noir. Naïve Production NV 826311, 2012 (CD).

Arthur H / Repac, Nicolas : L'or d'éros. Poésie sexuelle du 20e siècle. Nä̈ve NV830811 2014, spectacle-musical (CD).

Arthur H : Soleil dedans. Polydor 379329-6, 2014 (CD).

Barbara : Barbara chante Barbara. Philips B 77806 L, 1964 (33 tours).

Clerc, Julien : Double enfance. Virgin 09463374592 6, 2005 (CD).

Croze, Pauline : Pauline Croze. Wagram 3110542, 2005 (CD).

Debout sur le Zinc : Eldorado(s). Sarl DSLZ, 2015 (CD).

Dub Inc : So What. Diversité DIV36, 2016 (CD).

Frànçois and the Atlas Mountains : Solide mirage. Domino Records WIGCD379, 2017 (CD).

Halliday, Johnny: Que je t’aime. Philips 849468 BY, 1969 (33 tours).

IAM : Rêvolution. Def Jam Recordings France 5734027, 2017 (CD).

Lavilliers, Bernard : Clair-obscur. Barclay 537561-2, 1997 (CD).

Lavilliers, Bernard : Causes perdues et musiques tropicales. Barclay 275373 8, 2010 (CD).

L.E.J. : En attendant l'album. Universal 476 866-5, 2015 (CD).

Lenorman, Gérard: Olympia 75. Epic 25AP 247, 1975 (33 tours).

Manset, Gérard : Matrice. EMI 7930531, 1989 (33 tours).

Piaf, Edith : Exodus. Columbia CF 111, 1961 (45 tours).

Souchon, Alain : C'est déjà ça. Virgin 72438391782 2, 1993 (CD).

Tryo: Grain de sable. Salut Ô Productions YEL5112672, 2003 (CD).

Tryo : Ce que l'on sème. Salut Ô Productions 88697323462, 2008 (CD). 
Zebda : Le bruit et l'odeur. Barclay Productions 529222 2, 1995 (CD).

Zebda : Second tour. Barclay Productions 2792413, 2012 (CD). 